Supporting Information

\title{
Biocidal and Antifouling Chlorinated Protein Films
}

Li-Sheng Wang, $\dagger^{a}$ Akash Gupta, ${ }^{a}$ Bradley Duncan, ${ }^{a}$ Rajesh Ramanathan, ${ }^{b}$ Mahdieh Yazdani $^{a}$ and Vincent M. Rotello ${ }^{* a}$

${ }^{\mathrm{a}}$ Department of Chemistry, University of Massachusetts-Amherst, 710 N. Pleasant St., Amherst, MA 01003, USA.

${ }^{\mathrm{b}}$ Ian Potter NanoBioSensing Facility and NanoBiotechnology Research Laboratory, School of Applied Sciences, RMIT University, 124 La Trobe St, Melbourne VIC 3000, Australia

This supplementary information contains 7 pages and 6 figures. 


\section{Experimental Details}

Materials. Bovine serum albumin (BSA) was purchased from Fisher Scientific and used without further purification. Silica wafers were purchased from WRS Materials. Trichlor-O-Cide 5600 was purchased from Ecolab. DPD (N,N-diethyl-p-phenylenediamine) reagent was purchased from Fisher Scientific. MilliQ water was purified by using a Millipore water purification system.

Characterization. Atomic force microscopy was performed on a DI Dimension-3100 AFM. The average roughness of the determined sample area was calculated by Gwyddion, a freeware with the agreement of GNU General Public License. XPS analysis was performed on a Physical Electronics Quantum 2000 spectrometer using a monochromatic Al Ka excitation at a spot size of $10 \mathrm{~mm}$ with pass energy of 46.95. Chemically distinct species were resolved using a Gaussian Lorentzian function with nonlinear least-squares fitting procedure. All XPS spectra were background corrected using the Shirley algorithm and aligning the elemental binding energies to the adventitious carbon (C 1s) binding energy of $284.6 \mathrm{eV}$. The thickness of protein films was measured by a Rudolph Research Auto EL ellipsometer. Static water contact angle measurements were performed using a VCA Optima surface analysis/goniometry system with water droplets size of $2 \mu \mathrm{L}$. Scanning electron microscopic images were obtained by using FEI Magellan 400 field emission scanning electron microscope operated at $1 \mathrm{kV}$ with $13 \mu \mathrm{A}$ of beam current. Samples were coated with Au for 1 min before measuring.

Fabrication of protein films (BSA). $10 \% \mathrm{w} / \mathrm{w}$ solutions of BSA in MilliQ water were filtered by using a $0.22 \mu \mathrm{m}$ filter and spin-coated at $3000 \mathrm{rpm}$ for $30 \mathrm{~s}$ onto an oxygen plasma-cleaned silicon substrate, yielding a thin film of protein. Nanoimprinting of protein films was performed by using a Nanonex NX-2000 nanoimprinter with flat silicon molds. Imprinting was performed 
at $180{ }^{\circ} \mathrm{C}$ and $2.8 \mathrm{MPa}$ for $5 \mathrm{~min}$. All molds were treated with heptadecafluoro-1,1,2,2(tetrahydrodecyl)dimethyl-chlorosilane at $85{ }^{\circ} \mathrm{C}$ for 2 days in a vacuum chamber prior to use.

Fabrication of chlorinated protein film (Cl-BSA). BSA films were cut into $1 \mathrm{~cm}^{2}$ and immersed in $2 \mathrm{~mL}$ of $0.36 \mathrm{mg} / \mathrm{mL}$ of Trichlor-O-Cide 5600 solution in sample vials. After 24 hours, films were washed by Milli-Q water for 5 times and dry by nitrogen flow.

Release study of Cl-BSA film. Cl-BSA films were placed into $2 \mathrm{~mL}$ Milli-Q water in vials. After a period of time, the supernatants were collected for measuring the chlorine content, and replaced $2 \mathrm{~mL}$ of fresh Milli-Q water for the next period measurement. The concentration of chlorine in supernatants were determined by a $N, N$-diethyl- $p$-phenylenediamine (DPD) assay. $50 \mu \mathrm{L}$ of DPD reagent (prepared by mixing a foil packet of DPD total chlorine reagent powder (Hach, Loveland, CO) with $1 \mathrm{~mL}$ of DI water) were added into $2 \mathrm{~mL}$ of supernatants. The tubes were shaken for $5 \mathrm{~min}$ before measuring the absorbance at $512 \mathrm{~nm}$. Chlorine content of each sample was calculated by comparison to a standard curve prepared by Trichlor-O-Cide 5600 in water.

\section{Biocidal evaluation.}

Bacteria were inoculated in lysogeny broth (LB) medium at $37^{\circ} \mathrm{C}$ until stationary phase. The cultures were then harvested by centrifugation and washed with $0.85 \%$ sodium chloride solution three times. Concentrations of resuspended bacterial solution were determined by optical density measured at $600 \mathrm{~nm}$. M9 medium was used to make dilutions of bacterial solution to a concentration of $1 \times 10^{6} \mathrm{cfu} / \mathrm{mL}$. ${ }^{\mathrm{i}}$ Samples were then incubated in $2 \mathrm{~mL}$ of bacterial suspension for $24 \mathrm{~h}$ at $37^{\circ} \mathrm{C}$ at $275 \mathrm{rpm}$ in 12 -well culture plates. The optical densities of these solutions were then measured at $600 \mathrm{~nm}$ using a UV-vis Spectrometer. A growth control group without 
antimicrobial films and a sterile control group with only growth medium were carried out at the same time. Cultures were performed in triplicates, and at least two independent experiments were repeated on different days.

\section{Antifouling evaluation.}

Bacteria were inoculated in $3 \mathrm{~mL}$ LB broth and grown to stationary phase at $37^{\circ} \mathrm{C}$. The cultures were then diluted to O.D 0.1 in an M-9 media supplemented with $1 \mathrm{mM}$ IPTG (isopropyl $\beta$-D-1thiogalactopyranoside). ${ }^{\text {ii }} 2 \mathrm{~mL}$ of the dilution was poured onto the surfaces kept in 12 well culture plates. The surfaces were kept at $25^{\circ} \mathrm{C}$ and the bacteria were allowed to grow for 24 hours. In general, the surfaces with bacteria were rinsed in deionized water for three times before analysis under the microscope. 

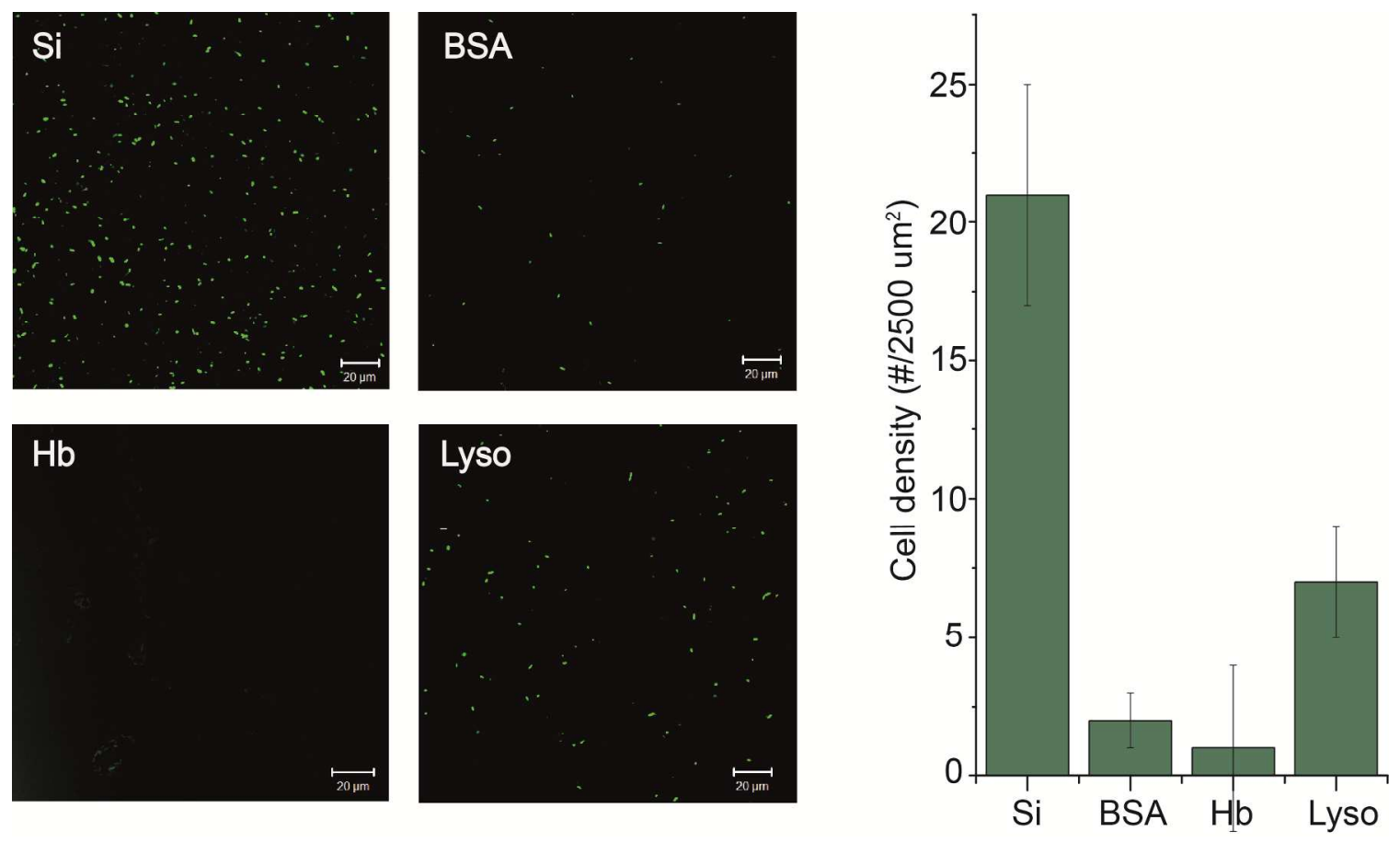

Figure S1. Adhesion of GFP expressed E. coli on silicon substrates and protein films after 24 hours incubation.

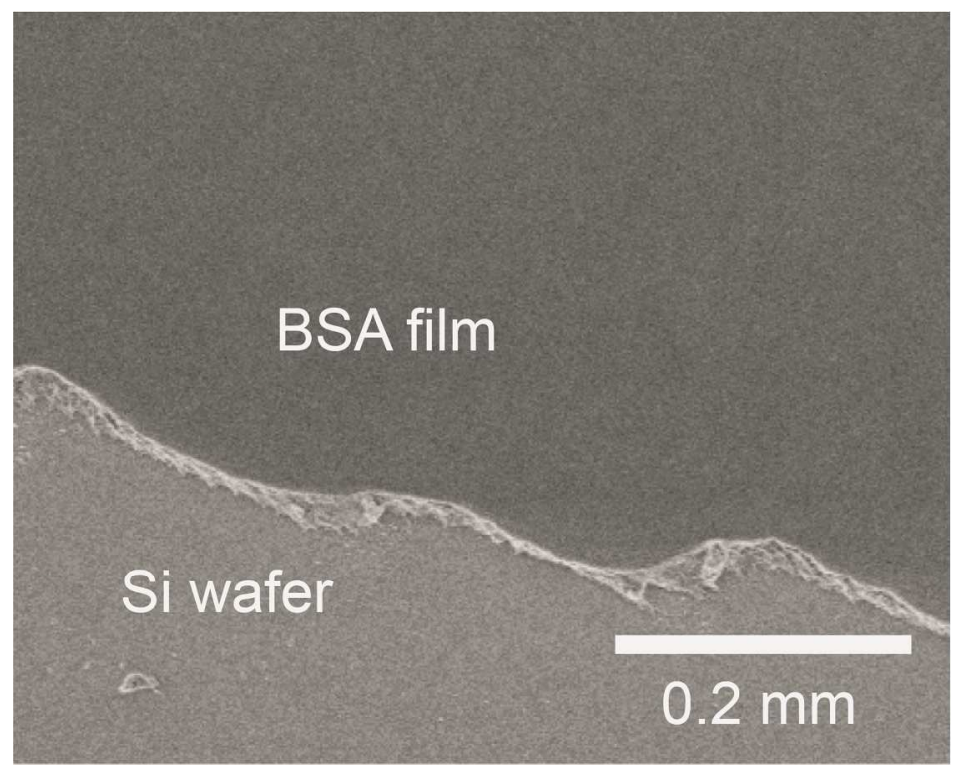

Figure S2. SEM image of scratched BSA film, showing a thin film with low surface roughness. 
(a)

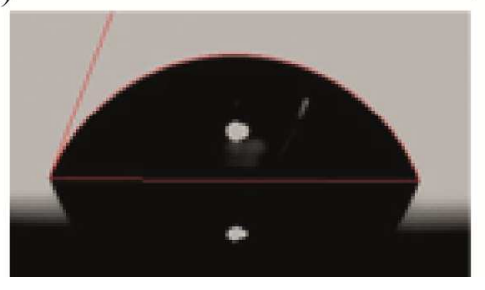

(b)

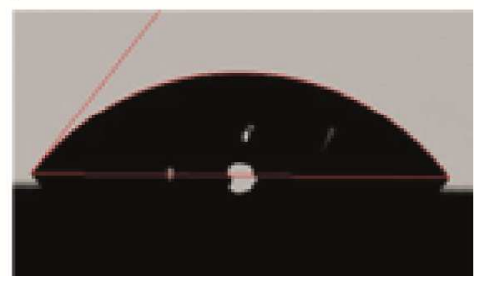

Figure S3. Optical images of contact angle measurements for (a) BSA and (b) Cl-BSA films.

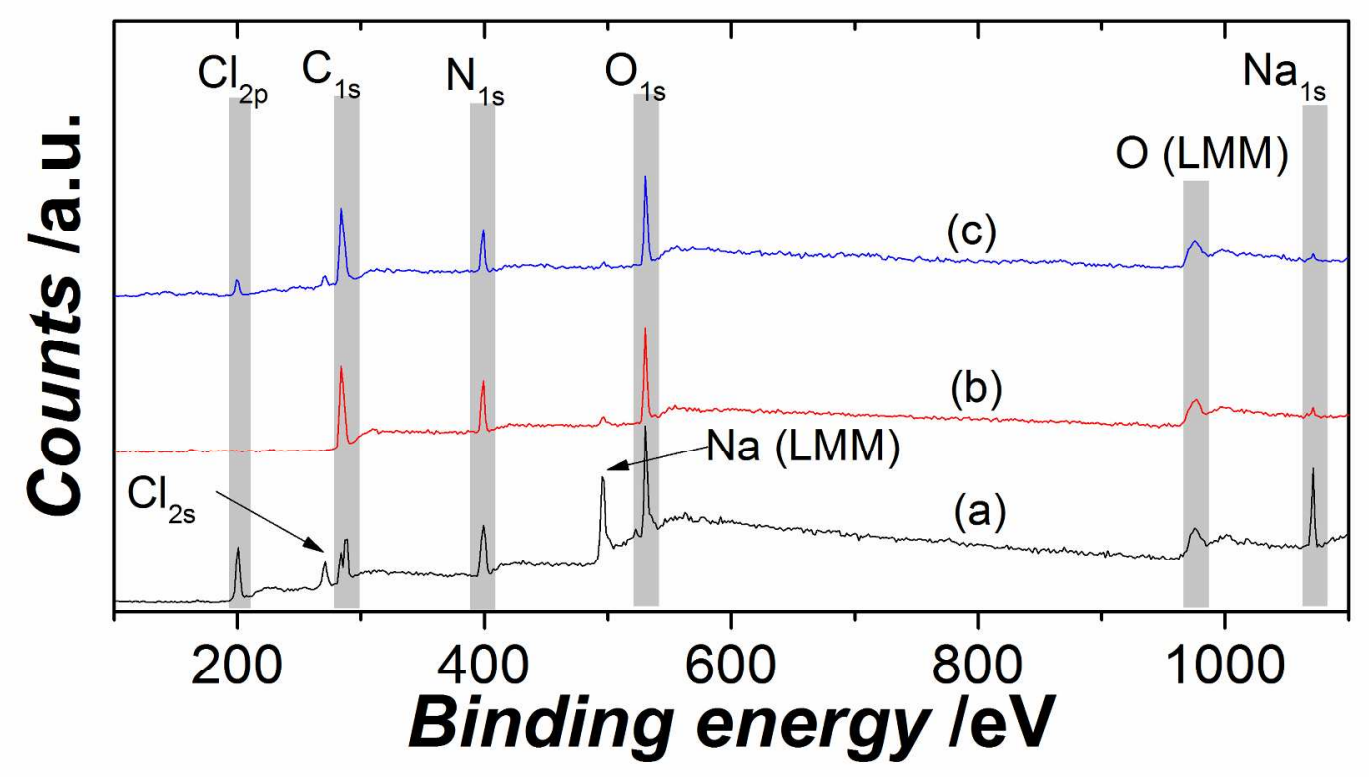

Figure S4. The survey spectrum obtained from (a) Trichlor-O-Cide 5600, (b) BSA film and (c) Cl-BSA film. The peaks corresponding to the respective elements have been marked. 

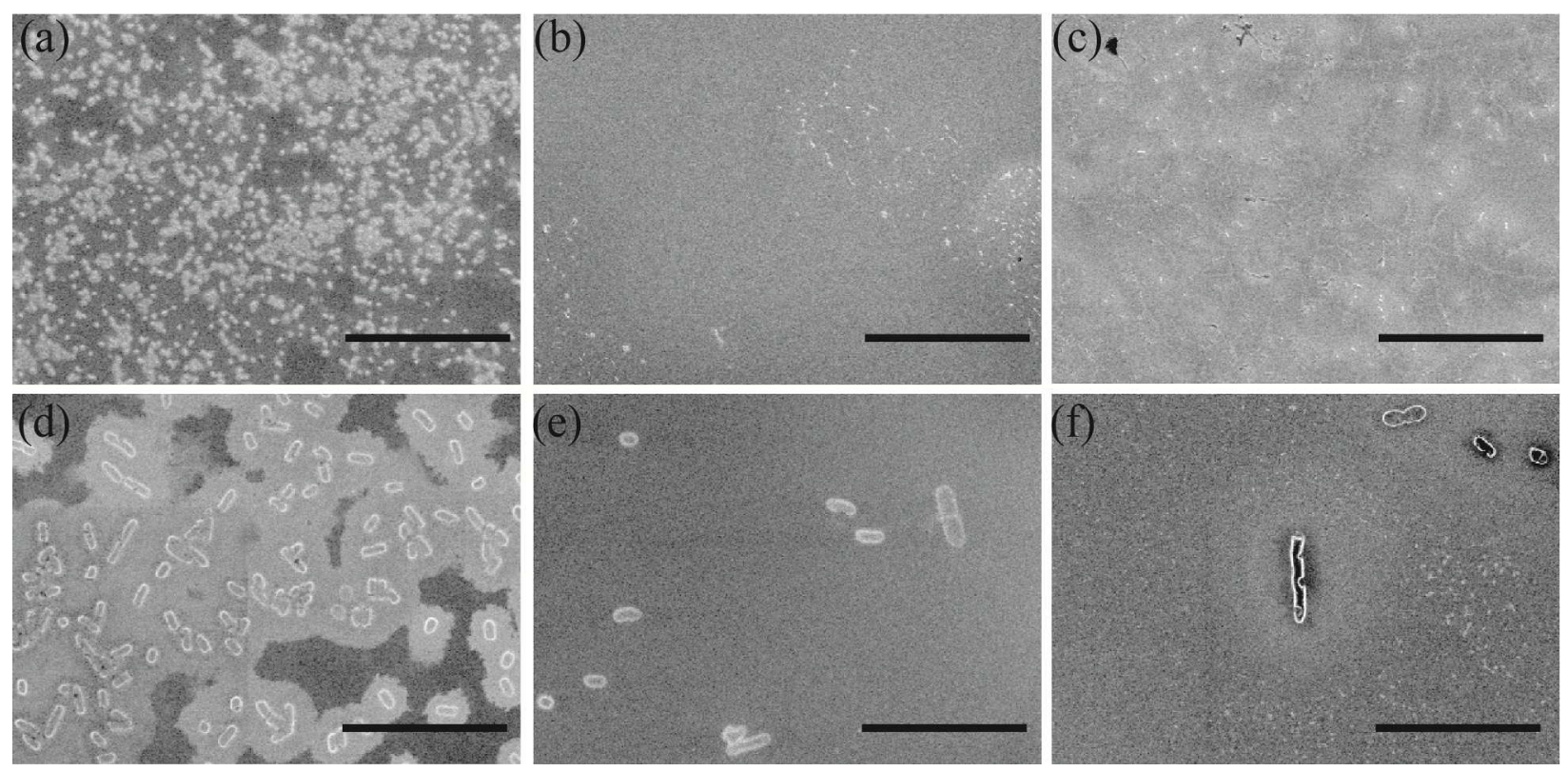

Figure S5. SEM images of E. coli adhered on $(a, d)$ silicon substrate, $(b, e)$ BSA film, and $(c, f)$

Cl-BSA film. Scale bars were $120 \mu \mathrm{m}$ for (a), (b), (c), and $12 \mu \mathrm{m}$ for (d), (e), (f).

(a)

(b)

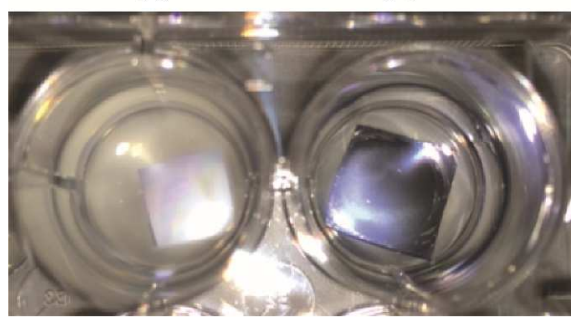

Figure S6. Optical images of E. coli incubating with (a) BSA (b) Cl-BSA films for 2 days.

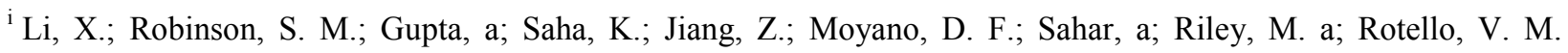
Functional gold nanoparticles as potent antimicrobial agents against multi-drug-resistant bacteria. ACS Nano 2014, 8 (10), 10682-10686 DOI: 10.1021/nn5042625.

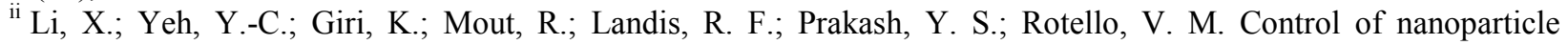
penetration into biofilms through surface design. Chem. Commun. 2015, 51, 282-285 DOI: 10.1039/C4CC07737G. 